potassium chlorides, and other salts, while Wohlgemuth ${ }^{1}$ supports this view with results from the study of some neutral salts, acids, amino acids and bases. On the other hand, Vernon ${ }^{2}$ has shown that salivary amylase acts much more slowly in the first few minutes of digestion than pancreatic amylase and points out differences in the action of several animal as well as plant amylases. Of course if the two enzymes are identical they must act similarly under all conditions, and apparent. differences in their action constitute stronger evidence than any observed similarities and until these differences are satisfactorily accounted for on some other basis, we can hardly do otherwise than consider the enzymes. distinct.

\title{
Conclusions.
}

Water softened by the use of lime was found to exert a pronounced inhibitory influence on the action of salivary and pancreatic amylases. This was due largely to the adsorption of the enzymes by colloidal magnesium hydroxide present in these softened waters.

That the two enzymes used in these tests, salivary and pancreatic amylases, are not identical, is indicated by differences shown in their response to the action of various ingredients contained in hard and softened: waters.

[From the LABORatory OF AGRICULTURAL CHEMISTRy OF THE UNTVERSTTY OF Wisconsin.]

\section{A SIMPLE METHOD FOR THE DETERMINATION OF CARBON IN ORGANIC MATERIALS.}

BY E. B. HART AND K. J. WOO.

Received June 26, 1913.

The determination of carbon in organic materials is still either'a laborious process or involves the use of expensive apparatus. Its determination by the Liebig or old combustion method is at present standard, but only in research or in special courses of instruction is it used. The more rapid method of carbon determination devised by $\mathrm{Parr}^{3}$ requires the use of $a$ bomb and the liberation of the gas under standard conditions for volumetric measurement. So far as we know this method has only been applied to carbon determinations in coal and soil. ${ }^{4}$ This method requires special apparatus which is somewhat expensive, although it gives accurate results.

The moist combustion methods which have been proposed for the determination of carbon in soils give low results. Incomplete oxidation

Biochem. Z., 9, I (1908).

2J. Physiol., 28, I56 (I902).

3 This Jolrnal, 26, 294 (I904).

${ }^{4}$ Pettit, Ibid., 26, 1640 (1904). 
by either the chromic acid or potassium permanganate methods indicates the unsatisfactory state of such procedures, although otherwise simple in operation.

The method here described was primarily developed for student use in the determinations of carbon in soil, but its ease of application and accuracy suggested a range of service which may give it wider application.

In the last year simple and accurate methods for the determination of carbon dioxide in carbonates have been devised, based upon the distillation of the carbon dioxide from an acid solution and its estimation by the double titration method, using phenolphthalein and methyl orange as indicators. A simple apparatus for this determination was perfected by Bowser ${ }^{1}$ and further modified by Brubaker. ${ }^{2}$

The essential principle of the method to be described, and which estimates the total carbon, consists in the oxidation of the carbon to carbon dioxide in an ordinary porcelain, nickel or iron crucible by means of sodium peroxide as already used by Parr and its fixation as a carbonate. The efficient oxidative powers of sodium peroxide are well known, but we were surprised to find that complete fixation of the carbon dioxide as carbonate could also be easily attained in an ordinary crucible. The second step in the process is the distillation of the carbon dioxide, its absorption in strong sodium hydroxide and its final estimation by the double titration process. We have worked with a considerable range of substances and with uniformly good results.

\section{Method.}

Approximately 6 grams of sodium peroxide are weighed out in an iron crucible of 40 to $50 \mathrm{cc}$. capacity and then 0 . I gram to 2 grams of the substance to be burned added. The crucible can be either of porcelain, nickel or iron, but should be of smooth, interior surface. Low results may be obtained in a porcelain crucible which has been excessively used and shows abrasion of the interior surface. The amount of sample to be taken will depend upon the amount of carbon present. For ordinary soils I to 2 grams should be used, but for peat soils or other organic substances with a carbon content greater than $40 \%$, the amount of sample should be reduced to 0.100 to $0.200 \mathrm{gram}$. When the carbon is low a small addition of mag. nesium, as recommended by Pettit, can be made for the purpose of accelerating the reaction. The charge is now well mixed, covered with a tight cover and set in another iron or nickel crucible of about I $20 \mathrm{cc}$. capacity and this outer crucible also covered. This is done for the purpose of preventing absorption of gases from the flame of the burner during the fusion. The further precaution of projecting the bottom of the crucible through a hole in an asbestos board should be followed. The mixture is next ignited

\footnotetext{
${ }^{1} J$. Ind. Eng. Chem., 4, 203 (1912).

2 Ibid., 4, 599 (1912).
} 
by applying the flame, regulating the heat so as to avoid too violent a reaction. This can generally be done by withdrawing the burner at the first sign of the beginning of the reaction. If the reaction proceeds with too great violence losses may occur and less sample should be taken. After the reaction has subsided the crucible should be heated for three to five minutes longer. The inner crucible and charge are next placed in a desiccator and allowed to cool. When cooled, the crucible is put in a 4 -inch funnel and the contents transferred to a $250 \mathrm{cc}$. flask with carbon dioxide free water. During this operation the funnel should be covered. with a watch glass. If the fusion has proceeded properly there will be no. residue of unburned carbon. The flask is next attached to the distilling: head, the solution made acid with $25 \%$ sulfuric acid and the carbon dioxide: distilled into the tower of beads, shown in the cut and to which has been.

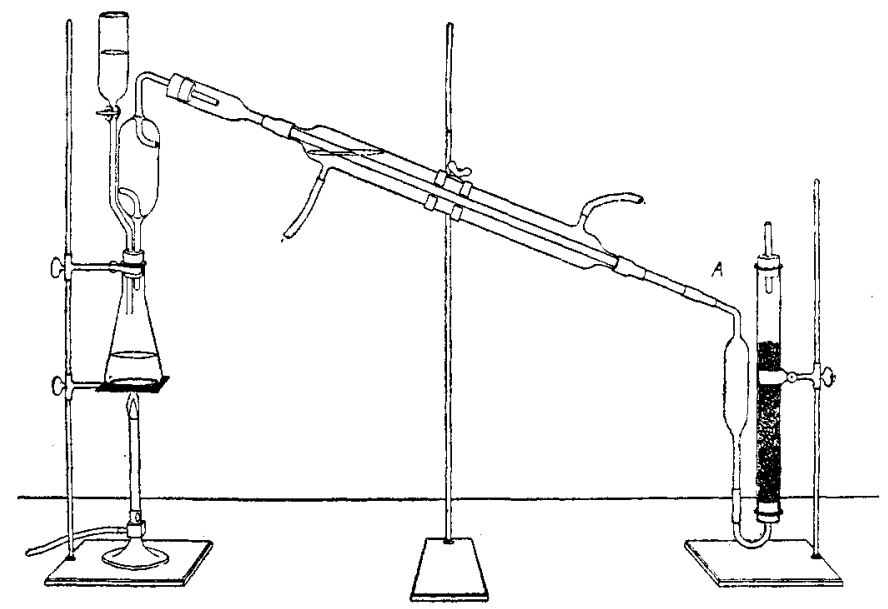

added ro cc. of 30 to $35 \%$ sodium hydroxide. The connections used in the construction of the tower are of rubber. The tower itself was made by cutting off the bottom of a test tube $35 \mathrm{~cm}$. long by $2.2 \mathrm{~cm}$. internal diameter.

This apparatus is the Bowser apparatus modified by placing a $50 \mathrm{cc}$. pipet between the condensor and the receiving tower. This prevents back suction and accomplishes in part what Brubaker sought to do with his. apparatus, but which in our hands was not satisfactory. After distilling. over about $60 \mathrm{cc}$. the apparatus is detached at $\mathrm{A}$ and the contents transferred to a $250 \mathrm{cc}$. flask by successive washings with small portions of carbon dioxide free water. This is easily accomplished by inclining the end of the pipet into the flask and pouring the wash water onto the beads. Complete washing should be tested for by placing a few drops of the water on a watch glass and adding phenolphthalein. It is always possible to. 
completely wash the tower and keep within the $250 \mathrm{cc}$. of volume. Phenolphthalein is next added to the solution and $25 \%$ sulfuric acid run in until the color begins to change. Care should be taken not to over-step the end point or low results may follow. The solution is now made up to $250 \mathrm{cc}$. and aliquots of $50 \mathrm{cc}$. taken for titration. A few drops more of phenolphthalein are added and the titration continued with o.I $N$ sulfuric acid to a neutral reaction. At this point and when the carbon exists as acid carbonate methyl orange is added and the titration continued.

With a little practice the inexperienced will have no trouble in getting a good end reaction, especially if a dilute solution of methyl orange is employed. The number of $\mathrm{cc}$. of o.I $N$ acid used during the titration with methyl orange as an indicator-minus the blank-multiplied by the carbon factor 0.0012 gives the weight of carbon in $50 \mathrm{cc}$. of the solution.

A correction blank is run in the same manner as above described to determin the carbon in the peroxide and the reagents used. This should be done on all fresh reagents. In materials such as soils, where both carbonates and organic carbon exist and where it is desired to know the proportion of each, the former can be estimated by the above method directly and before fusion, or by any other suitable method, and this result then subtracted from the total carbon obtained by the fusion process.

From the data ${ }^{1}$ in the table which follows, it will be noticed that in most cases the accuracy of the method has been extablished by the use of chemically pure materials. In some cases, as in soils, the method has been checked by the old combustion process. The sample of coal, with its carbon content, was kindly furnished us by Professor Parr of the University of Illinois.

We have also applied the method to urin and find that it works well. A measured volume or weight of urin-25 cc. - was placed in the crucible, made slightly acid and evaporated to half its volume to remove the carbon existing as carbonates. The mixture was then made slightly alkaline with sodium hydroxide and evaporated to dryness in a drying oven in order to aroid, as far as practical, absorption of carbon dioxide from the air. The dry residue was next mixed with sodium peroxide and the process carried out in the described manner.

The results recorded in the table are selected from a number of analyses made on each sample. Closer agreement was always secured on those materials low in carbon and when a sample larger than o.x gram could be taken. However, even on the materials high in carbon the method gave fairly accurate results.

${ }^{1}$ A part of the data reported in the table was collected by Mr. W. H. Juve in fulfilment of the requirements for the Bachelor's Degree at the University of Wisconsin, x913. 


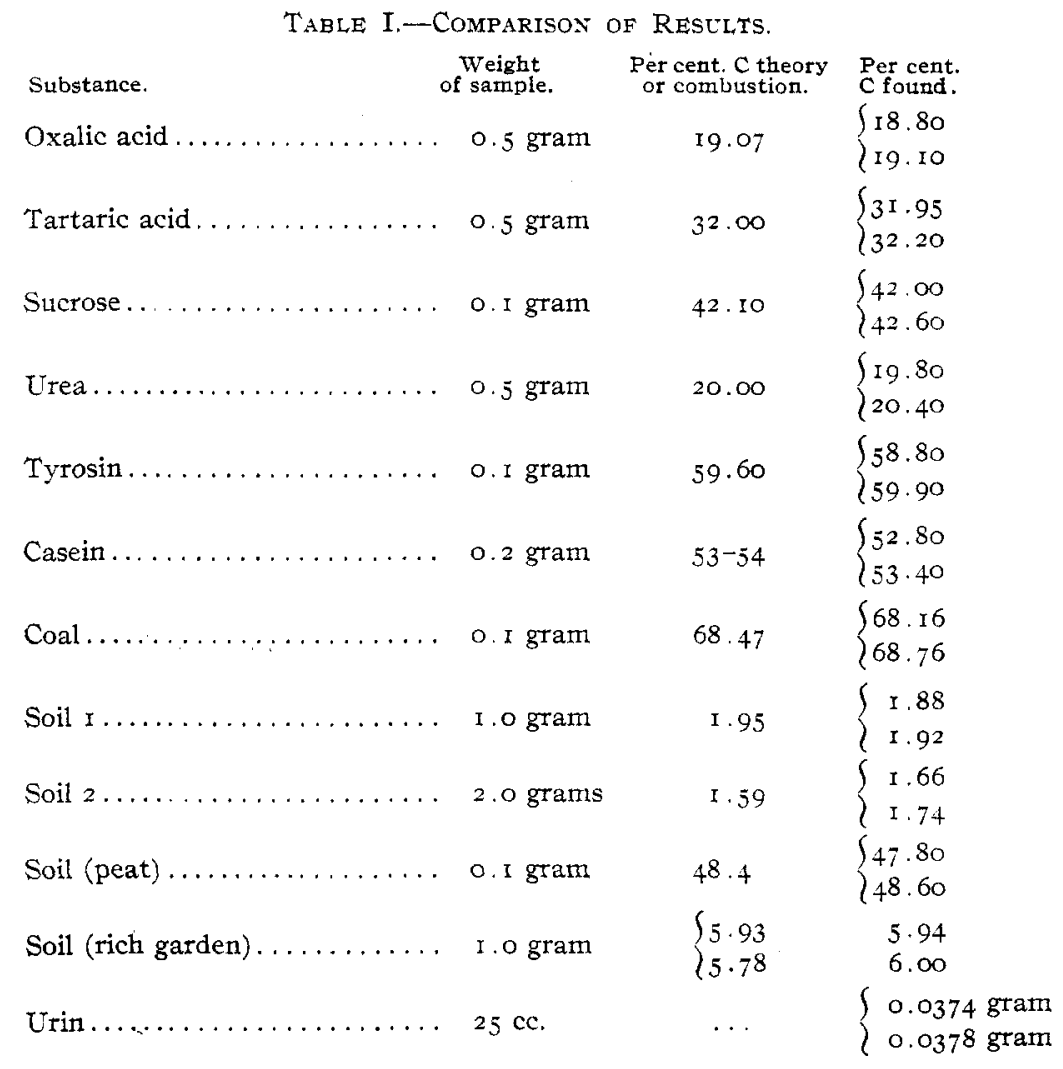

With reagents prepared and apparatus set up it is possible to complete a determination in half to three-fourths of an hour. The results in the hands of an experienced operator may be accurate to within one drop of o. I $N$ acid solution. For compounds high in carbon, small samples must be fused, otherwise there will be a loss of carbon by too violent an ignition. The possibility of error in compounds of this class will be somewhat greater than in compounds of lower carbon content, due to the small sample which must be taken.

This method may not, without some experience, be safe to use in place of the old combustion method for scientific research. But for routine analysis such as the carbon determination in soil, or as a substitute for the humus determination, which, in reality, has such little meaning, this method should undoubtedly find a place.

Further, in nutrition work where the effect of normal or abnormal rations are under investigation it makes possible the study of the carbon content of urin and feces and its relation to other constituents in the animal's excreta. 


\section{Summary.}

I. This paper describes a comparatively short and simple method for the determination of carbon in organic substances.

2. The principle of the method is the fusion of the material with sodium peroxide in an ordinary crucible whereby all of the carbon is converted into sodium carbonate.

3. The method gives accurate results and is especially suitable for routine carbon determinations in soils and also where it is desired to follow this element in certain phases of animal nutrition, as in urin analysis.

\section{NOTE.}

The Action of Silicon Tetrachloride on Aldehydes and Ketones.-A few years ago while trying to synthesize certain organo-silicon compounds, some silicon tetrachloride was added to pure, dry acetone. When examined a few hours later, the mixture had set to a jelly-like mass. The mass was broken up with water and a brown oil separated. This proved to consist chiefly of mesityl oxide, with probably small amounts of other condensation products of acetone.

As silicon tetrachloride is very unstable in the presence of water we concluded that water had been extracted from the acetone, resulting in the decomposition of silicon tetrachloride into silicic and hydrochloric acids, and the condensation of acetone into mesityl oxide. The reaction would be represented by the following equations:

$$
\begin{aligned}
& \mathrm{SiCl}_{4}+{ }_{4} \mathrm{H}_{2} \mathrm{O}=\mathrm{Si}(\mathrm{OH})_{4}+{ }_{4} \mathrm{HCl} . \\
& \mathrm{CH}_{3} \cdot \mathrm{CO} \cdot \mathrm{CH}_{3}+\mathrm{CH}_{3} \cdot \mathrm{CO} \cdot \mathrm{CH}_{3}=\left(\mathrm{CH}_{3}\right)_{2}: \mathrm{C}: \mathrm{CH} \cdot \mathrm{CO} \cdot \mathrm{CH}_{3}+\mathrm{H}_{2} \mathrm{O} .
\end{aligned}
$$

This reaction was tried out in a qualitative manner on several aldehydes and ketones and in all cases results analogous to the above were observed.

The silicon tetrachloride used in these experiments was prepared by passing hydrochloric acid gas over amorphous silicon, and fractionating the resulting mixture of silicon tetrachloride, silico-chloroform and probably other silicon chlorides. ${ }^{1}$ Silico-chloroform also has a condensing action on aldehydes and ketones.

To our knowledge, attention has never been called to this reaction. Circumstances have prevented a further study of its possibilities. As some of the condensation products of aldehydes and ketones are of considerable importance, it was thought that this reaction might be of sufficient interest to warrant this note.

JAMES N. CurRie.

Agricultural Experiment Station, Storrs, Conn.

${ }^{1}$ Besson and Fournier, Compt. rend., I48, 55.5 (1909). 